\title{
Utilização de benzodiazepínicos no Serviço Municipal de Saúde de Coronel Fabriciano, Minas Gerais
}

\author{
Use of benzodiazepines in local public health services \\ in Coronel Fabriciano in the State of Minas Gerais
}

Karleyla Fassarelo Firmino ${ }^{1}$

Mauro Henrique Nogueira Guimarães de Abreu ${ }^{2}$

Édson Perini ${ }^{1}$

Sérgia Maria Starling de Magalhães ${ }^{1}$

${ }^{1}$ Departamento de Farmácia Social, Faculdade de Farmácia, Universidade Federal de Minas Gerais. Av. Antônio Carlos 6627, Pampulha. 31270-901 Belo Horizonte MG. karleyla@superig.com.br ${ }^{2}$ Departamento de Odontologia Social e Preventiva, Faculdade de Odontologia, Universidade Federal de MInas Gerais
Abstract In this study, indications for benzodiazepines in the healthcare services of the city of Coronel Fabriciano (State of Minas Gerais, Brazil) were analyzed in terms of compliance with the indications established in the literature. The study was carried out using all prescriptions for benzodiazepines in municipal healthcare units between September and October 2006, as well as the prescription form filled out by the prescriber and computer files. A total of 1866 prescriptions were analyzed; $59.7 \%$ were for diazepam and the rest were for clonazepam. The mean daily dose per 1000 inhabitants/day was 24.69 for diazepam and 3.58 for clonazepam. Approximately 50\% of the indications were as a hypnotic or anti-anxiety medication; $21.9 \%$ were for "chronic use/dependence" and the rest were for other indications. Among the prescriptions that fulfilled the inclusion criteria for the analysis of indication adequacy (1618), approximately $70 \%$ were found to be inadequate considering the indication and duration of treatment. It revealed a high percentage of the inadequate use of benzodiazepines, especially due to prolonged use and the treatment of cases considered by the prescribers as chronic use/ dependence. Thus, healthcare services bear responsibility for the maintenance of dependence. Key words Benzodiazepine, Rational use, Diazepam, Health systems, Epidemiology
Resumo Neste estudo foram avaliadas as indicações de benzodiazepínicos no Serviço Municipal de Saúde de Coronel Fabriciano-MG, verificando sua conformidade com o preconizado pela literatura. O estudo avaliou todas as receitas desses medicamentos provenientes das Unidades Municipais de Saúde no período de Setembro a Outubro de 2006, os formulários de indicação clínica preenchidos pelo prescritor e cadastros informatizados do serviço. Analisaram-se 1.866 receitas, sendo 59,7\% do Diazepam e o restante do Clonazepam. A Dose Diária Definida por mil habitantes por dia foi de 24,69 para o Diazepam e de 3,58 para o Clonazepam. Cerca de 50\% das indicações relatadas pelos médicos foram como hipnótico ou ansiolítico, 21,9\% para "uso crônico/dependência" e o restante para outras indicações. Das receitas que atenderam aos critérios de inclusão para análise da adequação da indicação (1618), cerca de 70\% foram consideradas não adequadas, tendo em vista a indicação e o tempo de tratamento. Houve um alto percentual de inadequação na utilização de benzodiazepínicos, principalmente pelo uso prolongado e para atender a casos considerados pelos prescritores como uso crônico/dependência. Assim, há responsabilidade do serviço de saúde na manutenção da dependência.

Palavras-chave Benzodiazepínicos, Uso racional, Diazepam, Sistemas de saúde, Epidemiologia 


\section{Introdução}

Os benzodiazepínicos (BZD) são medicamentos hipnóticos e ansiolíticos com efeitos notáveis e com amplo índice terapêutico ${ }^{1}$. Apresentam, ainda, propriedades anticonvulsivante, relaxante muscular e amnésica ${ }^{1}$. O extenso uso inadequado dessa classe de medicamentos é relatado, tanto em países desenvolvidos quanto em desenvolvimento ${ }^{2-8}$.

Estima-se que cerca de 2\% da população adulta dos EUA e aproximadamente um milhão de pessoas no Reino Unido recebam uma prescrição de BZD por 12 meses ou mais, como ansiolítico ou hipnótico. Destes, aproximadamente $50 \%$ utilizam tais medicamentos por cinco ou mais anos ${ }^{9}$. No Chile, o uso inadequado dessa classe de medicamentos é reconhecido como um problema de saúde pública desde a década de 1980, quando foram detectadas prevalências alarmantes do consumo sem indicação clínica justificável ${ }^{6}$. No primeiro levantamento domiciliar nacional realizado no Brasil, em 2001, 3,3\% dos entrevistados declararam usá-los sem receita médica ${ }^{5}$. Estima-se que a população adulta usuária crônica de BZD seja igual a 1,6\%, e os indivíduos que abusam desses medicamentos geralmente o fazem para lidar com os problemas cotidianos e as reações de estresse da vida diária ${ }^{10}$.

$\mathrm{Na}$ Assistência Farmacêutica, além de garantir o acesso ao medicamento, as políticas de saúde devem criar mecanismos para acompanhar o uso, certificando de que o mesmo se dê segundo indicações clínicas definidas em evidências científicas e segundo as normas legais. O uso racional de medicamentos segundo a OMS ${ }^{11}$ consiste na utilização do medicamento apropriado às necessidades do paciente, na dose correta, por período de tempo adequado e a custo acessível. A ausência de atendimento a qualquer dos aspectos de racionalidade apontados nesse conceito implica em uso inadequado do medicamento.

O consumo de BZD em Coronel Fabriciano tem sido acompanhado pela Secretaria Municipal de Saúde (SMS) do município, por meio de relatórios mensais, desde 2002, detectando-se situações que suscitaram dúvidas quanto à adequação das indicações.

Este estudo teve como objetivo quantificar o consumo de benzodiazepínicos pela população usuária do Serviço Municipal de Saúde e avaliar a adequação das indicações clínicas desses medicamentos no município de Coronel Fabriciano - MG.

\section{Material e métodos}

O município de Coronel Fabriciano, localizado no Vale do Aço, na região leste do estado de Minas Gerais, apresentava, em 2004, população de 102.588 habitantes ${ }^{12}$. Conhecido como uma "cidade dormitório", suas atividades econômicas estão centradas na atividade industrial e no comércio, grande parte da população trabalhando nas atividades ligadas às siderúrgicas dos municípios vizinhos.

O Serviço Municipal de Saúde é composto por dez Unidades Básicas de Saúde (UBS), que contam com clínicos gerais, ginecologistas e pediatrias, um Centro de Especialidades (CEPS) responsável pela atenção secundária, com atendimento das especialidades médicas: cardiologia, oftalmologia, dermatologia, urologia, otorrinolaringologia, pneumologia e mastologia, duas equipes do Programa de Saúde da Família (PSF) e um Centro de Atenção à Saúde Mental (CASAM), com atendimento de neurologia e psiquiatria. Um hospital conveniado é responsável pela atenção no nível terciário.

A dispensação de medicamentos é feita nas farmácias das UBS, com exceção dos medicamentos psicotrópicos, dispensados na Farmácia Central. Em 2002 foi implantado um sistema informatizado para a dispensação de medicamentos. Por ocasião deste estudo, o sistema possuía cerca de 58 mil pacientes - usuários do Serviço Municipal de Saúde - cadastrados. A lista básica de medicamentos do município, em 2006, contava com 173 itens, incluindo o Diazepam $10 \mathrm{mg}$, Clonazepam $2 \mathrm{mg}$ e Clonazepam 2,5 mg/ml, dispensados mediante notificação de receita $\mathrm{B}$, de acordo com a legislação que aprova o regulamento técnico sobre substâncias e medicamentos sujeitos a controle especial - Portaria 344/98 SVS/MS 12 de maio de $1998^{13}$.

Trata-se de um estudo transversal com coleta retrospectiva de dados (setembro e outubro de 2006). Abrange o total das notificações de receita de BZD retidas na Farmácia Central, originadas nas unidades municipais de saúde (atenção primária e secundária). A escolha do período teve por objetivo evitar receitas de retorno do paciente ao serviço, pois a legislação estabelece a dispensação dos benzodiazepínicos, incluídos na lista $\mathrm{B}$, para um período máximo de sessenta dias de tratamento ${ }^{13}$.

Os dados foram coletados a partir das notificações de receita, do cadastro do paciente e do for- 
mulário de indicação clínica (FIC). Esse último documento foi instituído durante o estudo (agosto a outubro de 2006). Tratava-se de um formulário com campos abertos para preenchimento, pelo médico, do medicamento receitado e da indicação que justificava a prescrição. A indicação clínica presente no formulário foi preenchida pelo prescritor de forma aberta, com a utilização de termos técnicos, expressões da prática diária ou o Código Internacional de Doenças (CID - 10), de forma a não induzir o prescritor a nenhuma indicação clínica predefinida em campos fechados.

Foram incluídas no estudo para quantificação do consumo as notificações de receitas provenientes das unidades municipais de saúde, contendo o número de cadastro do paciente, acompanhadas dos FIC devidamente preenchidos. Se a notificação de receita não apresentava o número de cadastro do paciente, buscava-se localizá-lo pelo nome e, se este estivesse incompleto ou parcialmente ilegível, verificavam-se todos os cadastros com o mesmo nome para identificar qual paciente recebera prescrição naquela data. Foram excluídas as receitas de pacientes que não puderam ser identificadas e a segunda prescrição daqueles que retornaram à Farmácia Central durante o período do estudo. Foram também excluídos os casos de ausência, não preenchimento ou preenchimento incorreto ou ilegível do FIC. A coleta seguiu um protocolo estruturado contendo dados da notificação de receita (fármaco, concentração, posologia, duração do tratamento e especialidade do prescritor) e do FIC (indicação clínica do BZD). Foram acrescentados a idade, o gênero do paciente e o tempo de utilização contínua de BZD, acessado no sistema informatizado.

As indicações clínicas, coletadas dos FIC, foram agrupadas considerando a Classificação do CID-10 e a semelhança dos quadros descritos. Esse agrupamento foi definido por consenso entre os autores e por consultas a especialistas e à literatura ${ }^{1,14-18}$. Após agrupamento, o uso do BZD foi incluído nos seguintes subgrupos: como hipnótico, como ansiolítico e como de uso crônico/ dependência. Foram considerados termos equivalentes ao uso como hipnótico: transtorno ou distúrbio do sono; insônia; "não dorme sem ele"; "dificuldades para dormir". Síndrome do Pânico, Transtorno de Ansiedade Generalizada (TAG), Transtornos Fóbicos-Ansiosos, Transtorno Misto de ansiedade e depressão, agitação, irritabilidade e nervosismo foram agrupados como uso na ansiedade. As indicações clínicas descritas pelos médicos como "não vive sem ele", "não fica sem ele", "paciente que pediu”, "toma ou usa há muito tempo" foram agrupadas como Uso Crônico/Dependência. As demais indicações foram coletadas conforme descrito pelo prescritor.

Após esse agrupamento, as indicações clínicas foram lançadas em um formulário estruturado de coleta de dados. O formulário de coleta continha seis campos pré-definidos para a indicação e um campo aberto, nomeado de "outros". Desse modo as indicações foram enquadradas nas seguintes categorias:

1-Tratamento de transtornos do sono; 2Tratamento da ansiedade; 3- Uso crônico/ dependência; 4- Tratamento da depressão, 5- Tratamento de distúrbios convulsivos; 6- Tratamento da abstinência alcoólica; 7- Outros (coletado conforme descrito pelo prescritor).

Para a análise da adequação da indicação, foram excluídos os FIC com mais de uma indicação e as indicações com frequência menor que $2,5 \%$, pela dificuldade de agrupamento gerada por sua diversidade.

As indicações foram classificadas como adequadas ou inadequadas de acordo com os usos e o tempo de tratamento preconizados pela literatura $^{1,14-18}$. De acordo com a literatura, entre as principais indicações dos BZD na atenção primária estão: o uso no tratamento da ansiedade severa, insônia, epilepsia, espasmos musculares, síndrome de abstinência alcoólica, e como adjuvante no tratamento da esquizofrenia. Além disso, podem ser utilizados em crianças nos casos de terror noturno e sonambulismo ${ }^{1,14-18}$. Considerando essas indicações como norteadoras do uso de BZD, foi feita a análise da adequação da indicação. Assim, as indicações foram classificadas como inadequadas quando houve uso como hipnótico, ansiolítico, abstinência alcoólica, distúrbio neurovegetativo (DNV) por mais de seis meses consecutivos; todas as indicações agrupadas como uso crônico/dependência; indicação no tratamento da depressão sem prescrição concomitante de antidepressivo; e finalmente, as indicações não previstas na literatura de referência ${ }^{1,14-18}$.

Para a quantificação do consumo, feita em número de doses diárias definidas (DDD) por 1000 habitantes por dia, utilizaram-se os relatórios do sistema informatizado da Farmácia Central em 2006. Seguindo as recomendações da OMS, as DDD para o Diazepam $10 \mathrm{mg}$ (10 mg) e o Clonazepam $2 \mathrm{mg}$ ( $8 \mathrm{mg}$ ) foram aquelas preconizadas pela Anatomical Therapeutic Chemical Classification (ATC) ${ }^{19}$. O gasto com os BDZ foi calculado utilizando-se o número de unidades consumidas em 2006 e a média de preço unitário dos respectivos medicamentos. 
A coleta e análise dos dados ocorreram no período de 09/2006 a 07/2007.

A pesquisa foi conduzida dentro dos padrões da Declaração de Helsinki e aprovada pelo Comitê de Ética em Pesquisa da UFMG - COEP/ UFMG.

\section{Resultados}

De acordo com os relatórios do sistema informatizado, o consumo de BZD em 2006 totalizou 522.436 comprimidos de Diazepam $10 \mathrm{mg}$ e 303.629 comprimidos de Clonazepam $2 \mathrm{mg}$. Considerando-se a população cadastrada no sistema informatizado da Farmácia Central, o consumo foi de 24,69 e 3,58 DDD/1.000 habitantes/ dia, respectivamente, e um consumo total dos dois medicamentos de 28,37 DDD/1.000 habitantes/dia

Foram dispensados medicamentos prescritos em 2.113 receitas de BZD no período. Destas, $1.866(88,3 \%)$ atenderam aos critérios gerais de inclusão, sendo 59,7\% de Diazepam $10 \mathrm{mg}$ e 40,2\% de Clonazepam $2 \mathrm{mg}$. Uma única prescrição emitida de Clonazepam 2,5 mg/ml foi excluída da amostra.

Aproximadamente 75\% das prescrições destinavam-se a mulheres e indivíduos adultos, porém o número de idosos recebendo prescrição de BZD foi de aproximadamente 25\%. A média de idade foi 49,7 anos. A distribuição dos usuários de BZD segundo o gênero e faixa etária é apresentada na Tabela 1.

Do total de prescrições, $89,1 \%$ foram para 60 dias de tratamento. A posologia observada para $85,6 \%$ dos pacientes foi de um comprimido/dia, para ambos os fármacos (Tabela 2).

O tempo médio de tratamento contínuo observado foi superior a um ano (14,6 meses - 14

Tabela 1. Distribuição por gênero e faixa etária, de usuários de BZD do Serviço Municipal de Saúde de Coronel Fabriciano-MG, setembro/outubro/2006

\begin{tabular}{lrr}
\hline \multicolumn{1}{c}{ Variável } & n & \% \\
\hline Gênero & & \\
$\quad$ Feminino & 1387 & 74,3 \\
Masculino & 479 & 25,7 \\
Faixa Etária & & \\
$\quad<16$ anos & 4 & 0,3 \\
$16<$ idade $>60$ & 1412 & 75,7 \\
$>60$ anos & 450 & 24,1 \\
\hline
\end{tabular}

dias a 54 meses). A mediana foi de 9 meses, e o tratamento prolongado (maior de 6 meses) $\mathrm{Co}^{-}$ mum a mais de $50 \%$ dos pacientes (Tabela 2 ).

Clínicos gerais foram responsáveis pela emissão de $80,1 \%$ das prescrições, seguido pelos cardiologistas $(10,9 \%)$ e neurologistas $(4,3 \%)$. Psiquiatras foram responsáveis por apenas $0,2 \%$. Outras especialidades do Serviço Municipal de Saúde (ginecologia, pediatria, ortopedia, urologia, oftalmologia, dermatologia e pneumologia) prescreveram menos de $5 \%$ do total.

Após exclusão dos FIC que não atendiam aos critérios de inclusão para análise de adequação da indicação devido ao relato de mais de uma indicação ou indicações mais específicas com percentual inferior a 2,5\%,1618 FIC foram avaliados. Destaca-se que, excetuando-se o distúrbio neurovegetativo (DNV), as demais indicações classificadas como "outros" tiveram frequência inferior a 2,5\%. A maioria correspondia a indicações pouco sustentadas na literatura ou que necessitariam de outras informações do quadro do paciente e fugiam ao escopo deste estudo, justificando sua exclusão. Os usos como anticonvulsivante $(1,3 \%)$ e na abstinência alcoólica $(0,05 \%)$ também foram excluídos de acordo com o mesmo critério (Tabela 3 ).

Observou-se que quase $70 \%$ das indicações foram consideradas inadequadas. O uso como hipnótico e ansiolítico por tempo prolongado foi a indicação predominante, correspondendo a quase $50 \%$ das indicações inadequadas (Tabela 3 ).

Vale ressaltar que, após as exclusões, para os 1618 FIC analisados, as especialidades médicas

Tabela 2. Tempo de tratamento, posologia e duração do tratamento contínuo com benzodiazepínicos no Serviço Municipal de Saúde de Coronel Fabriciano-MG, setembro/outubro/ 2006

\begin{tabular}{lrr}
\hline \multicolumn{1}{c}{ Variável } & $\mathbf{n}$ & $\%$ \\
\hline Posologia & & \\
$\quad<1$ comprimido/dia & 57 & 3,0 \\
1 comprimido & 1.597 & 85,6 \\
$\quad>1$ comprimido/dia & 212 & 11,4 \\
Duração do tratamento & & \\
$\quad<60$ dias & 170 & 9,0 \\
$\quad 60$ dias & 1.663 & 89,1 \\
$>60$ dias & 33 & 1,8 \\
Duração de Tratamento Contínuo & & \\
$\quad$ 6 meses & 808 & 43,3 \\
$>6$ meses & 1.058 & 56,7 \\
\hline
\end{tabular}


Tabela 3. Indicação clínica e sua adequação para a prescrição de benzodiazepínicos no Serviço Municipal de Saúde de Coronel Fabriciano-MG, setembro/outubro/2006

\begin{tabular}{|c|c|c|}
\hline Variável & n (1618) & $\%$ \\
\hline \multicolumn{3}{|l|}{ Indicação Clínica } \\
\hline Hipnótico & 546 & 33,8 \\
\hline Ansiolítico & 392 & 24,2 \\
\hline Depressão & 159 & 9,8 \\
\hline Uso crônico / dependência & 354 & 21,9 \\
\hline Distúrbio Neurovegetativo (DNV) & 167 & 10,3 \\
\hline \multicolumn{3}{|l|}{ Adequação da indicação } \\
\hline Adequada & 509 & 31,5 \\
\hline Inadequada & 1.109 & 68,5 \\
\hline \multicolumn{3}{|l|}{ Condições de inadequação } \\
\hline $\begin{array}{l}\text { Uso prolongado como hipnótico } \\
\text { ou ansiolítico }\end{array}$ & 542 & 33,5 \\
\hline Uso crônico / dependência & 354 & 21,9 \\
\hline Distúrbio neurovegetativo & 167 & 10,3 \\
\hline $\begin{array}{l}\text { Uso na depressão sem } \\
\text { antidepressivo }\end{array}$ & 46 & 2,8 \\
\hline
\end{tabular}

emissoras sofreram poucas variações. O percentual de FIC emitido por neurologistas foi reduzido para $1,3 \%$. Os clínicos gerais permaneceram como os principais prescritores $(82 \%)$, seguido pelos cardiologistas $(10,9)$. O restante correspondeu às demais especialidades $(5,8 \%)$

Considerando-se o total de unidades dispensadas de BZD em 2006, estimou-se um gasto anual direto com a compra desses medicamentos em R $\$ 22.593,88$, correspondendo a 2,5\% dos gastos com medicamentos do município.

\section{Discussão}

A DDD é recomendada pela OMS para uso em estudos de utilização de medicamentos e representa a dose média diária do fármaco quando utilizado para sua principal indicação ${ }^{20}$.

A principal vantagem do emprego da DDD é a possibilidade de se fazer comparações entre locais ou tempos diferentes, sem que os resultados sejam comprometidos por variações de preço ou de apresentação. Contudo, é importante ressaltar que essa medida nem sempre equivale à dose média prescrita, ou ingerida.

Em fins dos anos 1980, Napo e Carlini estimaram o consumo de benzodiazepínicos no Brasil como 23,03 e 18,48 DDD/1.000 habitantes/dia como ansiolítico e hipnótico, respectivamente ${ }^{21}$. No decorrer dos últimos anos, apesar da melhora na difusão da informação e do acesso a novos medicamentos e tecnologias diagnósticas, o padrão de consumo parece ter se mantido. Ao contrário de outros medicamentos, que tendem a ser substituídos por novos agentes mais potentes ou mais seguros, o Diazepam (BZD de meiavida longa e notável efeito hipnótico), particularmente, tem se mantido entre os medicamentos mais populares em todo o mundo. O predomínio da prescrição do Diazepam foi também evidenciado em estudos no Brasil ${ }^{22,23}$, no Chile ${ }^{6}$, em $\mathrm{Cuba}^{24}$ e no Reino Unido ${ }^{25}$.

O predomínio de mulheres entre os usuários de benzodiazepínicos foi observado em vários estudos ${ }^{26,27} \mathrm{e}$ atribuído à maior preocupação das mulheres com a própria saúde e à busca por serviços de saúde ${ }^{26}$ e à maior prevalência da ansiedade e depressão entre elas ${ }^{28}$. Além desses fatores, Mastroianni et al. verificaram que, tanto para ansiolíticos como para antidepressivos, as propagandas de medicamentos utilizam majoritariamente figuras femininas, o que pode ter impacto direto sobre a prescrição ${ }^{29}$. Outra observação é que a condição de gênero feminino está associada à percepção médica distinta de necessidade de utilização de psicofármacos ${ }^{27,30}$.

Adultos corresponderam a cerca de $75 \%$ da amostra, porém o expressivo número de idosos entre os usuários confirma dados da literatura que apontam alta prevalência de utilização de BZD nessa faixa etária ${ }^{27,31}$.

No município não foi observada a prescrição expressiva de doses superiores à DDD. A posologia para mais de $80 \%$ dos pacientes foi de um comprimido de BZD por dia. Por outro lado, não se observaram significativamente doses inferiores à DDD, que poderiam indicar a adoção de protocolos de retirada do medicamento.

Verificou-se um alto consumo de BZD, que aparentemente se reforça com a tendência de prescrição das quantidades máximas permitidas pela regulamentação, estabelecida como o número de unidades posológicas necessárias para 60 dias de tratamento ${ }^{13}$.

A duração do tratamento com BDZ é uma das variáveis determinantes da racionalidade da terapia. Protocolos nacionais e internacionais ${ }^{14-17}$ consideram o tratamento superior a seis meses como de risco para o desenvolvimento de dependência e tolerância. No presente estudo, o uso de BZD por tempo prolongado (maior de 6 meses) atingiu mais de $50 \%$ dos pacientes, situação semelhante a outros relatos ${ }^{6,26,32}$. 
Os BZD, por serem medicamentos de amplo índice terapêutico ${ }^{1}$, boa tolerabilidade e baixo custo, trouxeram inegáveis benefícios no manejo de transtornos mentais, porém podem desencadear iatrogenias importantes, em grande parte geradas pelo uso inadequado. Deve-se considerar que os BZD são utilizados em situações clínicas pouco definidas no aspecto biomédico ${ }^{30}$. Suas principais indicações, insônia e ansiedade, são sintomas pouco específicos, comuns a várias doenças e intimamente relacionados à qualidade de vida. As dificuldades de caracterização do quadro clínico, associado à suposta segurança, contribuem para o uso abusivo desses medicamentos.

Observou-se que os clínicos gerais de Coronel Fabriciano foram os principais prescritores no município. O papel dos clínicos na prescrição de psicotrópicos tem sido descrito, tanto no Brasil quanto em outros países ${ }^{6,22,26,27,32}$. Esses profissionais, em geral, não se sentem confortáveis com essa situação e relatam dificuldade na abordagem de doenças mentais ${ }^{33}$, porém diante da dificuldade de estabelecer um diagnóstico e de encaminhar o paciente para um atendimento especializado, os clínicos tendem a instituir o tratamento e a prolongá-lo desnecessariamente. Não se pode ignorar ainda que a grande popularidade dessa classe de medicamentos é fortemente influenciada pela indústria farmacêutica, cujas propagandas enfatizam a segurança e transmitem a mensagem de que os psicofármacos, de acordo com Mastroianni et al. ${ }^{29}$, "tratam sintomas de desconforto do dia-a-dia induzindo um apelo irracional que pode se refletir na prescrição" 29

A cardiologia foi a segunda maior especialidade prescritora. A utilização de BZD por pacientes hipertensos está bem documentada na literatura ${ }^{24,27,34}$ e pode ter contribuído para o perfil de indicações observado.

Verifica-se um pequeno número de prescrições provenientes de psiquiatras, o que provavelmente se deve à ausência desses profissionais na rede de atendimento. As prescrições provenientes dessa especialidade se devem a atendimentos realizados por um psiquiatra da rede particular que, esporadicamente, atendia os casos graves na rede pública. A carência de psiquiatras no sistema público de saúde do município, para a adequada abordagem dos transtornos mentais, pode ser um fator importante relacionado à prescrição inadequada desses medicamentos.

Deve-se ressaltar que, neste estudo, toda a análise da adequação da indicação tem como base as informações do prescritor. Do ponto de vista do paciente, não necessariamente o uso se dá para a mesma indicação. Rozemberg, em inquérito de morbidades com lavradores do Espírito Santo, relata que entre as morbidades mais frequentes encontram-se os transtornos psiquiátricos designados genericamente pelos pacientes como doença dos nervos, que corresponde a queixas vagas enfatizando o discurso somático ${ }^{35}$. Silveira corrobora essas informações, a partir do relato de pacientes. Segundo a autora, a sintomatologia que define a "doença dos nervos" para o paciente é vaga e compromete várias funções orgânicas, compreendendo desde quadros leves de ansiedade até outros quadros graves de apatia e agressividade ${ }^{30}$.

Em Coronel Fabriciano, não foram pesquisados os relatos autorreferidos de morbidade que justificassem o uso do benzodiazepínico.

Considerando as indicações preconizadas pela literatura de referência ${ }^{1,14-18}$, a indicação clínica foi considerada inadequada em cerca de $70 \%$ das prescrições emitidas pelo Serviço Municipal de Saúde de Coronel Fabriciano.

Destacam-se as prescrições de benzodiazepínicos como hipnótico e ansiolítico por tempo prolongado. Embora essas sejam as principais indicações dos BZD, existe consenso na literatura de que seu uso deve se restringir a intervalo menor que seis meses ${ }^{14-18}$. Ainda que esse resultado se assemelhe a outros já descritos, reforçase a necessidade de intervenções para melhorar a condição de uso desses medicamentos.

A indicação clínica distúrbio neurovegetativo (DNV) apresentou uma frequência também elevada $(15,1 \%)$. O DNV é considerado uma disfunção somatoforme. Os sinais são semelhantes à sintomatologia de diversas doenças, particularmente do sistema cardiocirculatório, o que pode ser um fator de confusão, indutor de indicação clínica inadequada ${ }^{36}$. A abordagem desse quadro implica em uma propedêutica adequada, eliminando-se doenças de base e instituindose o acompanhamento psicoterapêutico. Quando necessário, a terapia medicamentosa específica deve ser instituída ${ }^{36}$. Se o BZD for empregado, deve-se utilizar o medicamento por período inferior a 6 meses. A elevada indicação de BZD para o distúrbio neurovegetativo é compatível com os trabalhos de Rozemberg ${ }^{35}$ e Silveira ${ }^{30}$, que relatam a tendência do paciente em associar queixas somáticas a problemas "de nervos". A inespecificidade das queixas e a diversidade de sintomas emocionais e orgânicos podem levar o médico a designar o quadro genericamente como DNV, justificando a prescrição do BZD.

Os BZD não apresentam efeito antidepressivo específico ${ }^{1}$ e sua prescrição no tratamento ini- 
cial da depressão é justificada, muitas vezes, pelo fato de depressão e ansiedade coexistirem. Porém, seu uso não associado ao antidepressivo é uma prática desaconselhada ${ }^{37}$. As indicações agrupadas na categoria "uso crônico/dependência" contribuíram com cerca de $20 \%$ das indicações. Essa observação contraria as indicações desses medicamentos e sugere que a dependência criada é sustentada pelo próprio sistema de saúde. Esse é um aspecto de grande relevância que pôde ser verificado neste estudo. Embora alguns autores relatem a pouca preocupação dos médicos e pacientes quanto aos riscos do uso crônico dos $\mathrm{BZD}^{2,5,10,38,39}$, o resultado observado aqui é surpreendente porque indica que os prescritores reconhecem a dependência já instalada, porém mantêm a prescrição, não instituindo medidas para reversão do quadro, uma vez que não se observou redução da dose do medicamento. Provavelmente, a confiança do prescritor na suposta segurança desses agentes terapêuticos, associada à pressão do paciente sobre o serviço de saúde e o temor de estabelecer um conflito com o paciente, sejam fatores que expliquem esse aspecto alarmante das indicações inadequadas. Em estudo realizado na Noruega, Dybwad et al. relatam que entre os fatores que levam clínicos a prescreverem benzodiazepínicos estão a dificuldade de negar a receita, o fato de os pacientes já usarem o medicamento devido a um diagnóstico prévio de outro profissional e o desejo de dar um alento à vida do paciente ${ }^{40}$. É possível que em Coronel Fabriciano esses mesmos fatores possam estar relacionados às indicações inadequadas dos BZD.

É importante ressaltar que o sistema de saúde pública do município não dispõe de profissionais e instrumentos de apoio ao dependente químico. A dependência é um quadro clínico que exige uma abordagem multidisciplinar para seu enfrentamento. A inexistência de terapias complementares, contando com outros profissionais de saúde, em espaço físico adequado, deixam o médico com poucas possibilidades de intervenção, reforçando a manutenção indefinida do tratamento. Paralelamente, o paciente não dispõe de esclarecimentos e alternativas para o enfrentamento das próprias angústias buscando na consulta médica e na prescrição do medicamento alívio para sua condição de sofrimento e legitimação social para sua condição de doente ${ }^{30}$.

Destaca-se que este estudo concentrou esforços na avaliação das indicações clínicas dos BZD, conforme a avaliação do prescritor, o que permitiu identificar aspectos relevantes para a caracterização da inadequação de sua indicação.
O alto índice de indicações inadequadas de BZD observado em Coronel Fabriciano, cidade de médio porte do interior do país, assemelha-se ao de grandes centros urbanos nacionais e do exterior $^{6,24,26,27,38,39,41}$, indicando um cenário globalizado de mau uso do medicamento.

Por outro lado, a carência de profissionais especializados, de opções de atenção psicoterapêutica e de medicamentos mais específicos disponíveis pelo sistema público de saúde do município podem ser aspectos mais locais, que possivelmente agravam o uso inadequado.

O uso irracional de BZD no município é preocupante e suas consequências são de difícil mensuração. Estudos permitem pontuar a possibilidade de agravos à saúde decorrentes dessa utilização, tais como quedas e fraturas, principalmente em idosos ${ }^{31,42-44}$, acidentes de trânsito ${ }^{45,46}$, intoxicações, suicídios ${ }^{47,48}$ e reações adversas proveniente de interações medicamentosas decorrentes da politerapia ${ }^{49}$, além do risco de dependência ${ }^{10}$.

Além de todas as implicações clínicas, o uso inadequado dos BZD tem ainda consequências farmacoeconômicas importantes. Aos valores diretos empregados no seu uso abusivo, somamse os recursos despendidos para contornar os problemas associados. Dos gastos com medicamentos em Coronel Fabriciano, em 2006, 2,5\% foram empregados na compra de BDZ, valor semelhante aos recursos gastos com medicamentos para condições crônicas de alta prevalência, como a hidroclorotiazida $(2,4 \%)$ e o propranolol (2,2\%). Considerando que a OMS estima que em 2020 os gastos com saúde mental deverão comprometer de $5 \%$ a $10 \%$ dos gastos com saúde, e que os recursos destinados à aquisição de medicamentos representam parte substancial dos custos de saúde, torna-se imprescindível a racionalização no uso dos agentes terapêuticos ${ }^{50}$.

Destaca-se que foram analisadas apenas as receitas emitidas pelo serviço público de saúde. Lima et al., em estudo em Botucatu- SP, verificaram que a renda per capita mostrou-se direta $\mathrm{e}$ independentemente associada ao uso de psicofármacos ${ }^{33}$, fazendo supor que os problemas relacionados ao uso de benzodiazepínicos sejam ainda mais graves no município se consideradas as prescrições emitidas pelos serviços particulares de saúde.

O contexto em que se dá o uso inadequado dos benzodiazepínicos é complexo e multifatorial. A instituição de protocolos clínicos bem elaborados, para subsidiar as decisões médicas, e a promoção de programas de atenção farmacêutica, ao usuário de BZD, esclarecendo o paciente 
quanto ao risco da utilização, podem ser ferramentas importantes para a redução do uso inadequado desses medicamentos e dos conflitos com o prescritor. A criação de serviços multiprofissionais de prevenção e apoio ao dependente de medicamentos e de centros de convivência para a atuação preventiva, além da contratação de médicos especialistas em saúde mental, são também medidas fundamentais para racionalizar o uso de benzodiazepínicos.

\section{Colaboradores}

KF Firmino, SMS Magalhães, E Perini e MHNG Abreu participaram igualmente de todas as etapas de elaboração do artigo.

\section{Referências}

1. Sweetman SC. Martindale: the complete drug refer-

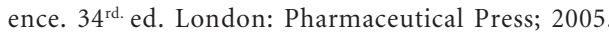

2. Diéye AM, Sylla M, Ndiaye M, Yoro Sy G, Faye B. Benzodiazepines prescription in Dakar: a study about prescribing habits and knowledge in general practitioners, neurologists and psychiatrists. Fundam Clin Pharmacol 2006; 20(3):235-238.

3. Furtado C, Teixeira I. Utilização de Benzodiazepinas em Portugal Continental (1999 - 2003). Acta Med Port 2006; 19(3):239-246.

4. Kassam A, Patten SB. Hypnotic use in populationbased sample of over thirty-five thousands interviewed Canadians. Popul Health Metr 2006; 4:15.

5. Noto AR, Orlandi P. Uso indevido de benzodiazepínicos: um estudo com informantes - chave no município de São Paulo. Rev Lat Am Enfermagem 2005; 13(N.Esp.):896-902.

6. Galleguillos T, Risco L, Garay JL, González M, Vogel M. Tendencia del uso de benzodiazepinas em una muestra de consultantes em atención primaria. Rev Med Chil 2003; 131(5):535-540.

7. Zandstra SM, Furer JW, Van De Lisdonk EH, Bor JHJ, Zitman FG, Van Weel C. Differences in health status between long-term and short-term benzodiazepine users. J Gen Pract 2002; 52(483):805-808.

8. Huf G, Lopes C.S, Rozenfeld S. O uso prolongado de benzodiazepínicos em mulheres de um centro de convivência para idosos. Cad Saude Publica 2000; 16(2):351-362.

9. Nomura K, Nakao M, Sato M, Yano E. Regular prescriptions for benzodiazepines: A Cross-Sectional study of outpatients at University Hospital. Intern Med 2006; 45(22):1279-1282.

10. Laranjeira R, Castro LAPG. Dependência de Benzodiazepínicos. Publicações dependência química [internet]. 2000. [acessado 2006 abr 08]. Disponível em: http:// www.uniad.org.br.

11. World Health Organization (WHO). The rational use of drugs. Report of the conference of experts. Nairobi: World Health Organization (WHO);1987. 
12. Instituto Brasileiro de Geografia e Estatística (IBGE). População do município de Coronel Fabriciano - MG. Coronel Fabriciano. [Internet]. 2004. [acessado 2006 abr 08]. Disponível em: http://www.fabriciano. mg.gov.br.

13. Brasil. Ministério da Saúde (MS). Agência Nacional de Vigilância Sanitária. Portaria 344 - Regulamento o uso de medicamentos sujeitos a controle especial. [Internet]. 1998 [acessado 2007 nov 03]. Disponível em: http://www.e-legis.anvisa.gov.br

14. Brasil. Ministério da Saúde (MS). Secretaria de Assistência à Saúde. Departamento de Assistência e Promoção à Saúde. Coordenação Saúde Mental. $O$ uso dos psicofármacos na clínica. Brasília: Ministério da Saúde (MS); 1994.

15. O'Brien CP. Benzodiazepines and substance abuse. Academic highlights - Benzodiazepines: Revisiting Clinical Issues in Treating Anxiety Disorders. Prim Care Companion J Clin Psychiatry 2005; 7(1):23-32.

16. The Royal Australian College of General Practioners (RACGP) Guidelines. Guideline for benzodiazepine. The Royal Australian College of General Practioners. [Internet] 2000. [acessado 2007 ago 16]. Disponível em: http:// www.racgp.org.au/guidelines

17. Ford C, Kay R, Barjolin JC. Guidance on Prescribing Benzodiazepines to Drugs users in Primary Care. Substance Misuse Management in General Pratice. [internet]. 2005. [cited 2007 Aug 20]. Available from: http://www.smmgp.org.uk.

18. United States Pharmacopeia Dispensing Information. USP-DI Advice for the Patient Drug Information in Lay Language. 23rd. ed. Massachusetts: Micromedex; 2003.

19. World Health Organization (WHO). WHO Collaborating Centre for Drug Statistics Methodology. History of the ATC/DDD. Oslo: Norwegian Institute of Public Health; 2007.

20. Melo DO, Ribeiro E, Storpirtis S. A importância e a história dos estudos de utilização de medicamentos. Rev. Bras. Cienc. Farm 2006; 42(4):475-485.
21. Napo SA, Carlini EA. Benzodiazepínicos no Brasil: um perfil do consumo nos anos de 1988 e 1989 . J Bras Psiquiatr 1993; 42(6):313-319.

22. Sebastião ECO, Pelá IR. Consumo de psicotrópicos: análise das prescrições ambulatoriais como base para estudo de problemas relacionados com medicamentos. Seguim Farmacoter 2004; 2(4):250-257.

23. Noto AR, Carlini EA, Mastroianni PC, Alves AC, Galduróz JCF, Kuroiwa W, Csizmar J, Costa A, Faria MA, Hidalgo SR, Assis S, Nappo SA. Análise de prescrições e dispensação de medicamentos em duas cidades no estado de São Paulo, Brazil. Rev Bras Psiquiatr 2002; 24(2):68-73.

24. Herrera LS, Fabián EV, Alós IY, Freijoso E. Utilización de benzodiazepinas en la Atención Primaria de Salud. Rev Cubana Med Gen Integr 2002; 18(3):187-190

25. Craig D, Passmore P, Fullerton KJ, Beringer TRO, Gilmore DH, Crawford VLS, Mccaffrey PM, Montgomery A. Factors influencing prescription of CNS medications in different elderly populations. Pharmacoepidemiol Drug Saf 2003; 12(5):383-387.

26. Andrade MF, Andrade RCG, Santos V. Prescrição de psicotrópicos: avaliação das informações contidas em receitas e notificações. Braz J Pharm Sci 2004; 40(4):471-479.

27. Rodrigues MAP, Facchini LA, Lima MS. Modificações nos padrões de consumo de psicofármacos em localidade do sul do Brasil. Rev Saude Publica 2006, 40(1):107-114.

28. Kinrys G, Wygant LE. Transtornos de ansiedade em mulheres: gênero influência o tratamento? Rev Bras Psiquiatr 2005; 27(Supl. 2):S43-50.

29. Mastroianni P C, Vaz ACR, Noto AR, Galduróz JCF. Análise do conteúdo de propagandas de medicamentos psicoativos. Rev Saude Publica 2008; 42(5):968-971.

30. Silveira ML. O Nervo Cala, O Nervo Fala: A Linguagem da Doença. Rio de Janeiro: Fiocruz; 2000. 
31. Passaro A, Volpato S, Romagnoni F, Manzoli N, Zuliani, G, Fellin R. Benzodiazepines with different half-life and falling in a hospitalized population: the GIFA study. J Clin Pharmacol 2000; 53(12): 1222-1229.

32. Poyares D, Pinto Júnior LR, Tavares S, Vieira SB Hipnoindutores e insônia. Rev Bras Psiquiatr 2005; 27(1):2-7.

33. Lima MCP, Menezes PR, Carandina L, Cesar CLG, Barros MBA, Goldbaum M. Transtornos mentais comuns e uso de psicofármacos: impacto das condições socioeconômicas. Rev Saude Publica 2008; 42(4):717-723.

34. Srisurapanont M, Garner P, Critchley J, Wongpakaran N. Benzodiazepine prescribing behaviour and attitudes: a survey among general practitioners practicing in northern Thailand. BMC Fam Pract 2005 $6: 27$.

35. Rozemberg B. O consumo de calmantes e o "problema de nervos" entre lavradores. Rev Saude Publica 1994; 28(4):300-308

36. Bombana JA, Leite ALSS, Miranda CT. Como atender aos que somatizam? Descrição de um programa e relatos concisos de casos. Rev Bras Psiquiatr 2000; 22(4):180-184.

37. National Institute Clinical Excellence (NICE). De pression: Management of depression in primary and secondary care. [Internet]. 2004. [acessado 2007 ago 16]. Disponível em: http://www.nice.org.uk/CG023

38. Cook JM, Marshall R, Masci C, Coyne JC. Physicians' Perspectives on prescribing benzodiazepines for older adults: a qualitative study. I Gen Intern Med 2007; 22(3):303-307.

39. Barter G, Cormack M. The long - term use of benzodiazepines: patients'views, accounts and experiences. Farm Pract 1996; 13(6):491-497.

40. Dybwad TB, Eskerud J, Laerum E. Why are some doctors high prescribers of benzodiazepines and minor opiates? A qualitative study of GPs in Norway. Fam Pract 1996; 14(5):361-368.

41. Del Pozo JG, Iglesias FJA, Garcia-Pando AC, Corominas DM, Sanz MM, Del Pozo VG. Utilización de ansiolíticos e hipnóticos em Espana (1995 2002). Rev Esp Salud Publica 2004; 78(3):379-387.
42. Homero GE, Marin PPL, Castro SH, Trinidad HM, Valenzuela EA. Caídas em adultos mayores institucionalizados: Descripción y evaluación geriátrica. Rev Med Chil 2003; 131(8):887-894.

43. Coutinho ESF, Silva SD. Uso de medicamentos como fator de risco para fratura grave decorrente de queda em idosos. Cad Saude Publica 2002; 18(5):1359-1366.

44. Chaimowicz F, Ferreira TJXM, Miguel DFA. Use of psychoactive drugs and related falls among older people living in a community in Brazil. Rev Saude Publica 2000; 34(6):631-635.

45. Barbone F, Mcmahon AD, Davey PG, Morris AD, Reid IC, Mcdevitt DG, Macdonald TM. Association of road-traffic accidents with benzodiazepine use. Lancet 1998; 352(9137):1324-1325.

46. Verster JC, Veldhuijzen DS, Patat A, Olivier B, Volkerts ER. Hypnotics and Driving Safety: Meta-Analyses of Randomized Controlled Trials Applying the on-the-road Driving Test. Curr Drug Saf 2006; 1(1):63-71.

47. Bortoletto ME, Bochner R. Impacto dos medicamentos nas intoxicações humanas no Brasil. Cad Saude Publica 1999; 15(4):859-869.

48. Romão MR, Vieira LJES. Tentativas suicidas por envenenamento. Rev Bras Prom Saude 2004; 17(1): 14-20.

49. Juurlink DN, Mandani M, Kopp A, Laupacis A, Redelmeier DA. Drug - Drug interactions among elderly patients hospitalized for drug toxicity. JAMA 2003; 289(13):1652-1658.

50. World Health Organization (WHO). Department of Mental Health and Substance Abuse. Economic aspects of the mental health system. Key messages to health planners and policy-makers. Genebra: World Health Organization (WHO); 2006.

Artigo apresentado em 09/11/2009

Aprovado em 10/02/2010

Versão final apresentada em 26/02/2010 